\title{
A Theoretical Study of the Tautomerism and Vibrational Spectra of 4,5-Diamine-2,6-dimercaptopyrimidine
}

\author{
R. A. Timm, ${ }^{a}$ J. A. Bonacin, ${ }^{a}$ A. L. B. Formiga ${ }^{b}$ and H. E. Toma ${ }^{*, a}$ \\ aInstituto de Química, Universidade de São Paulo, CP 26077, 05513-970 São Paulo-SP, Brazil \\ ${ }^{b}$ Instituto de Química, Universidade Estadual do Rio de Janeiro, Rio de Janeiro-RJ, Brazil
}

\begin{abstract}
A 4,5-diamina-2,6-dimercaptopirimidina (DADMcP) constitui uma molécula multifuncional que apresenta uma tautomeria bastante complexa, com nove formas isoméricas possíveis. O estudo da tautomeria nesse composto foi realizado por meio da espectroscopia FTIR em associação com cálculos ab-initio HF/SCF e DFT. De acordo com os resultados teóricos, três tautômeros são favorecidos energeticamente, sendo a tiona a forma a mais estável. Os espectros vibracionais das formas tautoméricas mais estáveis foram simulados através de cálculos de DFT, permitindo a atribuição e elucidação do quadro complexo de bandas vibracionais observado experimentalmente, envolvendo a mistura de isômeros.
\end{abstract}

The 4,5-diamine-2,6-dimercaptopyrimidine (DADMcP) compound is an interesting multifunctional species exhibiting a rather complex tautomerism, encompassing nine tautomeric forms. Investigation of tautomerism in this compound has been carried out by means of FTIR spectroscopy, in association with ab-initio HF/SCF and DFT calculations. According to this study three tautomers are energetically favored; the thione form being the most stable one. The theoretical vibrational spectra of such tautomeric forms have been successfully simulated by means of DFT calculations, allowing the elucidation and assignment of the complex composition of the vibrational bands observed for the mixture of isomers.

Keywords: mercaptopyrimidines, DFT calculations, tautomerism, spectral simulation

\section{Introduction}

Mercaptopyrimidines are interesting compounds, which have been widely studied over the past few years ${ }^{1}$ because of their many interesting applications, especially in coordination chemistry. By incorporating both $\mathrm{S}$ and $\mathrm{N}$ atoms in their structures, they are able to bind transition metal ions, acting as monodentate ${ }^{2}$ and more frequently as chelating and bridging ligands. ${ }^{3-5}$ These compounds exhibit tautomeric equilibrium between the thiol ( $>\mathrm{C}$-SH) and thione ( $>\mathrm{C}=\mathrm{S}$ ) forms, as a consequence of the highly mobile protons in their structure. ${ }^{6-8}$ In fact, thione-thiol tautomeric equilibrium have attracted great experimental ${ }^{9-13}$ and theoretical interest ${ }^{14-17}$ in chemistry and biochemistry. These forms can mutually interchange via intramolecular proton transfer between the nitrogen and the nearby carbonyl sulfur. The interchange mechanism is solvent dependent; usually, the thione form predominates in polar

*e-mail: henetoma@iq.usp.br solvent while the thiol is favorable in gas phase or nonpolar solvents. . $^{14,16,18,19}$

In this article, we present a theoretical study on the tautomerism of 4,5-diamine-2,6-dimercaptopyrimidine (DADMcP). This compound is an interesting multifunctional species capable of undergoing electrochemical polymerization and of sequestering transition metal ions. ${ }^{20}$ Experimental support for the existence of the tautomeric forms of this molecule was obtained from FTIR spectroscopy, in analogy with a recent work on violuric acid and 6-amino-5-nitrouracil. ${ }^{21}$ Further insight into the molecular structures was provided by theoretical calculations using ab-initio Hartree-Fock Self-Consistent Field (HF/SCF) and the density functional theory (DFT) approaches.

\section{Experimental}

The compound 4,5-diamine-2,6-dimercaptopyrimidine was obtained from Aldrich. FTIR spectra of the solid 
samples in the range of 4,000-400 $\mathrm{cm}^{-1}$ were recorded on a Shimadzu FTIR-8300 spectrophotometer, using $\mathrm{KBr}$ pellets. The NIR spectra in the range of $29,000-4,000 \mathrm{~cm}^{-1}$ were recorded on a FieldSpec fiber optics instrument from Analytical Spectral Devices (ASD).

Computational calculations were carried out using the GAMESS(R4) software ${ }^{22}$ for geometry optimization. In all cases, a convergence criterion of $10^{-4} \mathrm{kcal}^{-1} \mathrm{~mol}^{-1}$ was used in a conjugate gradient algorithm. Molecular orbitals were expanded using the atomic 6-311G(d,p) basis set to solve the Hartree-Fock (HF) equations as well as the Kohn-Sham ones (DFT). The gradient-corrected density functional methodology employed in DFT calculation was the B3LYP hybrid. ${ }^{23-25}$ The harmonic vibrational frequencies and intensities were calculated at the same levels of theory with the analytical evaluation of second derivatives of energy as a function of atomic coordinates and the calculated intensities were utilized to generate the theoretical spectra. Low and high frequencies were scaled by factors of 1.01 and 1.06, respectively, and zero-point energies by a factor of 0.9806 , as recommended by Scott and Radom. ${ }^{26}$

The theoretical vibrational spectra shown in Figure 3, were calculated from the sum of Gaussian shaped bands, based on the equation: ${ }^{24}$

$T(\omega)=2,174 \times 10^{8} \sum_{\mathrm{I}} \frac{f_{I}}{\Delta_{1 / 2}} \exp \left(-2,773 \frac{\left(\omega-\omega_{I}\right)^{2}}{\Delta_{1 / 2}^{2}}\right)$

where $\mathrm{T}$ is transmittance, and assuming half-bandwidths $\left(\Delta_{1 / 2}\right)$ of $40 \mathrm{~cm}^{-1}$. The sum in the equation includes all allowed vibration transitions with energies, $\omega_{\mathrm{I}}$ (expressed in $\mathrm{cm}^{-1}$ ), and oscillator strengths, $f_{\mathrm{I}}$ which were obtained from the theoretical calculations. The total integrated intensity is equal to the sum of the oscillator strengths:

$$
4,319 \times 10^{-9} \int \varepsilon(\omega) d \omega=\sum_{I} f_{I}
$$

\section{Results and Discussion}

Theoretical investigation of DADMcP compound showed nine possible tautomers, here denoted 1 to 9 (Figure 1 ). The geometry of each one was optimized by $a b$-initio RHF/SCF and DFT methods. More than one conformer was found for each tautomer, and the most stable conformer was chosen for further analysis. The energies of each tautomer are shown in Table 1. The usefulness of DFT methods to deal with the tautomerism of nucleic acid bases is a rather well established point. In particular, vibrational frequencies and zero-point energies have been estimated<smiles>Nc1nc([Hg])nc(C[Hg])c1N</smiles><smiles>[2H]N([2H])C1=C(N)N(P)C(=S)NC1=C</smiles>

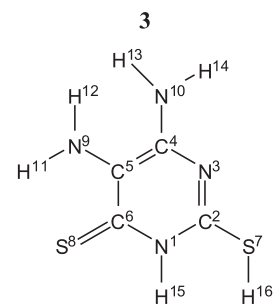<smiles>Nc1[nH]c(S)nc(=S)c1N</smiles><smiles>Nc1[nH]c(=S)nc(S)c1N</smiles><smiles>Nc1nc(=S)[nH]c(S)c1N</smiles><smiles>N=c1[nH]c(S)nc(S)c1N</smiles><smiles>N=c1nc(S)[nH]c(S)c1N</smiles><smiles>N=c1[nH]c(=S)[nH]c(S)c1N</smiles>

Figure 1. Tautomeric forms of 4,5-diamine-2,6-dimercapto-pyrimidine.

very accurately, and frequently with errors smaller than those reported using MP2 theory ${ }^{27-29}$. According to HF/SCF and DFT calculations, the order of stability is $\mathbf{2}>\mathbf{3}>\mathbf{1}>$ $\mathbf{6}>\mathbf{9}>\mathbf{5}>\mathbf{4}>\mathbf{7}>\mathbf{8}$. The dithione form is expected to be the most stable one (tautomer $\mathbf{2}$ ).

Based on these results, the vibrational spectra of the three most stable tautomers of $\operatorname{DADMcP}(\mathbf{1}, \mathbf{2}$ and $\mathbf{3})$ were calculated theoretically, as shown in Figure 3. In fact, the DFT frequencies provided a good insight into the vibrational structure of the three tautomeric species, as shown in Tables 2-4.

Table 1. HF/SCF, DFT and Zero-point theoretical energies ( $\left.\mathrm{kcal} \mathrm{mol}^{-1}\right)$ for the 4,5-diamine-2,6-dimercapto-pyrimidine tautomers (vacuum) and energy difference with respect to tautomer 2

\begin{tabular}{ccccccc}
\hline Tautomer & $\mathrm{HF} / \mathrm{SCF}$ & $\Delta \mathrm{E}$ & $\mathrm{DFT}$ & $\Delta \mathrm{E}$ & $\mathrm{ZPE}$ & $\Delta \mathrm{E}^{\mathrm{ZPE}}$ \\
\hline $\mathbf{1}$ & $-732,895.2$ & 5.8 & $-734,976.5$ & 9.7 & 66.6 & 4.6 \\
$\mathbf{2}$ & $-732,901.0$ & 0 & $-734,986.2$ & 0 & 71.7 & 0 \\
$\mathbf{3}$ & $-732,896.4$ & 4.5 & $-734,980.3$ & 5.9 & 69.3 & 3.5 \\
$\mathbf{4}$ & $-732,875.2$ & 25.8 & $-734,960.8$ & 25.4 & & \\
$\mathbf{5}$ & $-732,882.2$ & 18.7 & $-734,967.8$ & 18.4 & & \\
$\mathbf{6}$ & $-732,886.5$ & 14.5 & $-734,970.4$ & 15.8 & & \\
$\mathbf{7}$ & $-732,871.4$ & 29.6 & $-734,954.7$ & 31.5 & & \\
$\mathbf{8}$ & $-732,864.5$ & 36.4 & $-734,950.1$ & 36.1 & & \\
$\mathbf{9}$ & $-732,884.7$ & 16.2 & -734.969 .8 & 16.4 & & \\
\hline
\end{tabular}

Zero-point vibrational energies for the three tautomers confirmed the stability order, but the energy gap is large enough to indicate tautomer $\mathbf{2}$ should predominate in gas phase. However, for this tautomer, the comparison of the theoretical and the experimental FTIR spectrum of the 
DADMcP species (Figure 3) shows that the number of bands in the experimental spectrum is higher than for the predicted one, specially in the $400-1700 \mathrm{~cm}^{-1}$ region. For this reason, the occurrence of a mixture of tautomers seems indeed rather plausible in the solid state.

The experimental infrared spectrum of DADMcP is shown in Figure 2, revealing a rather complex composition of the vibration bands for this compound. In a first approximation, the group of broad bands from 3600 to 2800 $\mathrm{cm}^{-1}$ can be attributed to $v_{\mathrm{as}}(\mathrm{N}-\mathrm{H})$ and $v_{\mathrm{s}}(\mathrm{N}-\mathrm{H})$ of the free and hydrogen bonded molecules in the solid. The shoulders around $2600 \mathrm{~cm}^{-1} \mathrm{can}$ be tentatively ascribed to $v(\mathrm{~S}-\mathrm{H})$. The bands at 1565 and $1647 \mathrm{~cm}^{-1}$ can be attributed to $\delta(\mathrm{N}-\mathrm{H})$ in $\mathrm{NH}_{2}$ groups. The bands at 1300 and $1346 \mathrm{~cm}^{-1}$ can be assigned to $v(C=C)+v(C=N)$ vibrations. The remaining bands are associated with amine groups and aromatic ring vibrations.

A correlation study, trying to match each pair of experimental and theoretical vibrational band, has been carried out, as shown in Tables 2-4. Tautomer 1 exhibits a

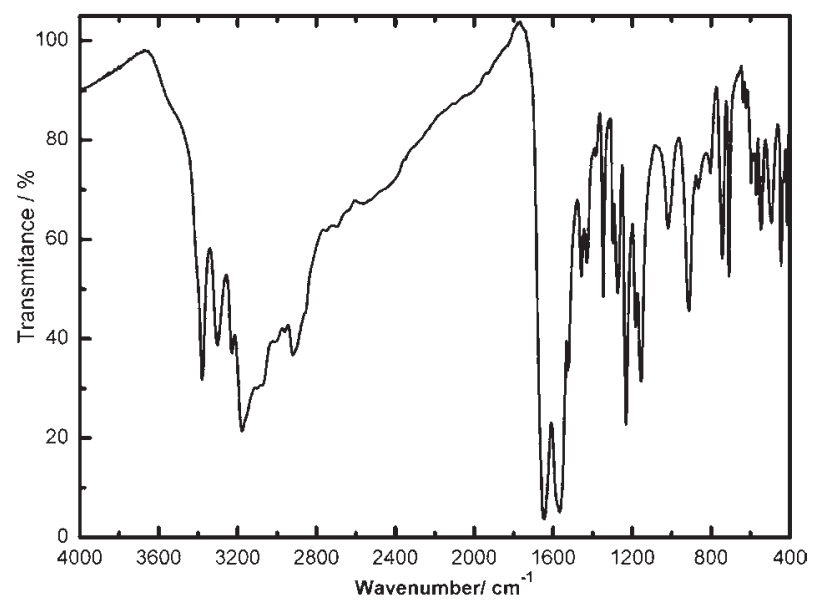

Figure 2. FTIR spectrum of the 4,5-diamine-2,6-dimercaptopyrimidine compound ( $\mathrm{KBr}$ pellets).

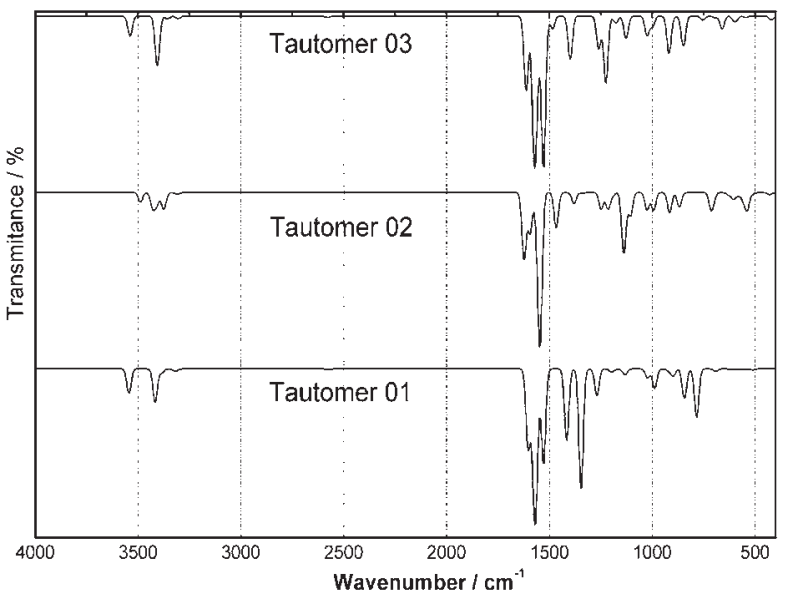

Figure 3. Theoretical FTIR spectra of the DADMcP tautomers 1, 2 and $\mathbf{3}$.
Table 2. Experimental and theoretical vibrational spectrum* of tautomer 1

\begin{tabular}{|c|c|c|c|}
\hline Mode & $\begin{array}{l}\text { Exp. / } \\
\mathrm{cm}^{-1}\end{array}$ & $\begin{array}{l}\text { Theor. / } \\
\mathrm{cm}^{-1}\end{array}$ & Assignments \\
\hline 42 & 3565 & 3521 & $\nu_{\text {as }} \mathrm{N}_{10} \mathrm{H}_{13,14}[98.5]$ \\
\hline 41 & 3379 & 3387 & $v_{\mathrm{s}} \mathrm{N}_{10} \mathrm{H}_{13,14}[98.8]$ \\
\hline 40 & 3300 & 3319 & $\mathrm{v}_{\mathrm{as}} \mathrm{N}_{9} \mathrm{H}_{11,12}[99.4]$ \\
\hline 39 & 3230 & 3261 & $v_{\mathrm{s}} \mathrm{N}_{9} \mathrm{H}_{11,12}[99.1]$ \\
\hline 38 & 2693 & 2678 & $v_{8} \mathrm{H}_{15}[97.3]$ \\
\hline 37 & 2577 & 2662 & $v S_{7} H_{16}[97.3]$ \\
\hline 36 & 1647 & 1648 & $\begin{array}{l}\delta \mathrm{N}_{10} \mathrm{H}_{13,14}[44.0]+\delta \mathrm{N}_{9} \mathrm{H}_{11,12}[42.1]+ \\
v \mathrm{~N}_{3} \mathrm{C}_{4,5}[9.5]\end{array}$ \\
\hline 35 & 1565 & 1626 & $\delta \mathrm{N}_{9} \mathrm{H}_{11,12}[57.1]+\delta \mathrm{N}_{10} \mathrm{H}_{13,14}[36.4]$ \\
\hline 34 & 1525 & 1577 & $\begin{array}{l}\left(v \mathrm{v}_{4,5,6}+\delta \mathrm{C}_{4} \mathrm{~N}_{10} \mathrm{H}_{13}+v\left(\mathrm{C}_{5}-\mathrm{N}_{9} \mathrm{H}_{11,12}\right)[70.3]+\right. \\
v \mathrm{~N}_{3} \mathrm{C}_{4,5}[16.7]+\delta \mathrm{S}_{8} \mathrm{H}_{15}[8.3]\end{array}$ \\
\hline 33 & 1462 & 1541 & $\begin{array}{l}\delta \mathrm{N}_{10} \mathrm{H}_{13,14}[30.3]+\delta \mathrm{N}_{9} \mathrm{H}_{11,12}[28.6]+ \\
v \mathrm{C}_{4,5,6}[19.3]+v \mathrm{~N}_{3} \mathrm{C}_{4} \mathrm{~N}_{1}[16.5]+ \\
\delta \mathrm{S}_{7} \mathrm{H}_{16}[3.5]\end{array}$ \\
\hline 32 & 1430 & 1471 & $\begin{array}{l}\left(\mathrm{vC}_{4} \mathrm{~N}_{10}+\delta \mathrm{N}_{10} \mathrm{H}_{13,14}\right)[61.3]+\left(\mathrm{vC}_{5,6}+\right. \\
\mathrm{v}\left(\mathrm{C}_{5}-\mathrm{N}_{19} \mathrm{H}_{11,12}\right)[18.7]+v \mathrm{~N}_{3} \mathrm{C}_{2} \mathrm{~N}_{1}[12.1]\end{array}$ \\
\hline 31 & 1346 & 1370 & $\begin{array}{l}\left(v \mathrm{C}_{4,5,6}+\mathrm{v}\left(\mathrm{C}_{5}-\mathrm{N}_{9} \mathrm{H}_{11,12}\right)[45.4]+\right. \\
v \mathrm{~N}_{3} \mathrm{C}_{2} \mathrm{~N}_{1}[34.2]+\delta \mathrm{N}_{10} \mathrm{H}_{13,14}[14.6]\end{array}$ \\
\hline 30 & 1300 & 1294 & $\begin{array}{l}\left(v \mathrm{C}_{6} \mathrm{~N}_{1} \mathrm{C}_{2}+v \mathrm{C}_{2} \mathrm{~S}_{7}\right)[45.8]+v \mathrm{~N}_{3} \mathrm{C}_{4,5}[21.3]+ \\
\delta \mathrm{N}_{10} \mathrm{H}_{13,14}[16.2]+\delta \mathrm{N}_{19} \mathrm{H}_{11,12}[12.2]\end{array}$ \\
\hline 29 & 1276 & 1270 & $\begin{array}{l}\rho \mathrm{N}_{10} \mathrm{H}_{13,14}[40.9]+v \mathrm{C}_{6} \mathrm{~N}_{1} \mathrm{C}_{2}[22.9]+ \\
v \mathrm{~N}_{3} \mathrm{C}_{4,5}[17.5]+\delta \mathrm{N}_{9} \mathrm{H}_{11,12}[8.4]+ \\
\delta \mathrm{S}_{8} \mathrm{H}_{15}[8.1]\end{array}$ \\
\hline 28 & 1232 & 1218 & $\begin{array}{l}v\left(\mathrm{C}_{5}-\mathrm{N}_{9} \mathrm{H}_{11,12}\right)[31.7]+\rho \mathrm{N}_{10} \mathrm{H}_{13,14}[31.1]+ \\
v \mathrm{C}_{6} \mathrm{~N}_{1} \mathrm{C}_{2}[19.7]+\delta \mathrm{S}_{8} \mathrm{H}_{15}[8.8]+v \mathrm{~N}_{3} \mathrm{C}_{6}[5.8]\end{array}$ \\
\hline 27 & 1182 & 1153 & $v \mathrm{~N}_{9} \mathrm{H}_{11,12}[92.4]$ \\
\hline 26 & 1152 & 1084 & $\begin{array}{l}\rho \mathrm{N}_{10} \mathrm{H}_{13,14}[55]+\mathrm{vN}_{3} \mathrm{C}_{4} \mathrm{~N}_{10}[13.8]+ \\
\delta \mathrm{S}_{8} \mathrm{H}_{15}[10.1]+\delta \mathrm{S}_{7} \mathrm{H}_{15}[5.8]+v \mathrm{C}_{4,5,6}[9.2]\end{array}$ \\
\hline 25 & 1015 & 1028 & $\begin{array}{l}\delta \mathrm{S}_{8} \mathrm{H}_{15}[43.9]+\left(\mathrm{vC}_{4,5,6}+\mathrm{vC}_{4}-\mathrm{N}_{10} \mathrm{H}_{13,11}\right) \\
{[28.8]+v \mathrm{~N}_{3} \mathrm{C}_{2} \mathrm{~N}_{1}[15.3]+\delta \mathrm{N}_{9} \mathrm{H}_{11,12}[8.9]}\end{array}$ \\
\hline 24 & & 981 & $\begin{array}{l}\delta \mathrm{S}_{8} \mathrm{H}_{15}[45,3]+\rho \mathrm{N}_{10} \mathrm{H}_{13,14}[17.0]+ \\
v \mathrm{C}_{6} \mathrm{~N}_{1} \mathrm{C}_{2}[13.9]+\delta \mathrm{S}_{7} \mathrm{H}_{16}[12.6]+ \\
v \mathrm{~N}_{5} \mathrm{C}_{4,5}[7.9]\end{array}$ \\
\hline 23 & 910 & 917 & $\begin{array}{l}\delta \mathrm{S}_{7} \mathrm{H}_{16}[59.5]+\delta \mathrm{S}_{8} \mathrm{H}_{15}[11.9]+ \\
v \mathrm{C}_{6} \mathrm{~N}_{1} \mathrm{C}_{2}[10.7]+\rho \mathrm{N}_{10} \mathrm{H}_{13,14}[9.6]+ \\
v \mathrm{~N}_{5} \mathrm{C}_{4,5}[4.5]\end{array}$ \\
\hline 22 & 863 & 863 & 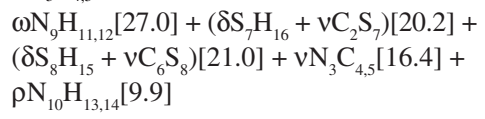 \\
\hline 21 & 806 & 790 & $\begin{array}{l}\omega \mathrm{N}_{9} \mathrm{H}_{11,12}[72.8]+\rho \mathrm{N}_{10} \mathrm{H}_{13,14}[9.5]+ \\
v \mathrm{~N}_{3} \mathrm{C}_{4,5}[8.2]+\delta \mathrm{S}_{7} \mathrm{H}_{16}[3.0]\end{array}$ \\
\hline 20 & 746 & 782 & $\operatorname{\tau ring}[56,6]+\tau \mathrm{N}_{9} \mathrm{H}_{11,12}[34,5]$ \\
\hline 19 & 712 & 718 & $\begin{array}{l}\omega \mathrm{N}_{9} \mathrm{H}_{11,12}[32.4]+\text { ring breath[35.4] }+ \\
\rho \mathrm{N}_{10} \mathrm{H}_{13,14}[20.9]+\delta \mathrm{S}_{8} \mathrm{H}_{15}[11.1]\end{array}$ \\
\hline 18 & 639 & 681 & $\begin{array}{l}\tau \text { ring }[52.3]+\tau \mathrm{N}_{10} \mathrm{H}_{13,14}[30.5]+ \\
\tau \mathrm{N}_{9} \mathrm{H}_{11,12}[17.1]\end{array}$ \\
\hline 17 & 621 & 629 & $\begin{array}{l}\left.\tau \mathrm{N}_{10} \mathrm{H}_{13,14}[75.4]+\rho \mathrm{N}_{9} \mathrm{H}_{11,12} 12.4\right]+ \\
\tau \operatorname{ring}[10.2]\end{array}$ \\
\hline 16 & 600 & 608 & tring $[60.7]+\rho \mathrm{N}_{9} \mathrm{H}_{11,12}[27.1]$ \\
\hline 15 & 571 & 577 & $\begin{array}{l}\text { Ring breath[38.6] }+\rho \mathrm{N}_{10} \mathrm{H}_{13,14}[27.7]+ \\
\omega \mathrm{N}_{9} \mathrm{H}_{11,12}[19.4]+\left(\delta \mathrm{S}_{8} \mathrm{H}_{15}+\delta \mathrm{S}_{7} \mathrm{H}_{16}\right)[14.7]\end{array}$ \\
\hline 14 & 545 & 545 & $\begin{array}{l}\rho \mathrm{N}_{10} \mathrm{H}_{13,14}[31.3]+\delta \operatorname{ring}[28.9]+ \\
\omega \mathrm{N}_{9} \mathrm{H}_{11,12}[21.1]+\left(\delta \mathrm{S}_{8} \mathrm{H}_{15}+\delta \mathrm{S}_{7} \mathrm{H}_{16}\right)[18.6]\end{array}$ \\
\hline 13 & 446 & 408 & $\delta$ ring \\
\hline 12 & 412 & 395 & ring breath \\
\hline
\end{tabular}

*Fractional composition of the vibrational modes are shown in parenthesis. 
Table 3. Experimental and theoretical vibrational spectrum of tautomer 2

\begin{tabular}{|c|c|c|c|}
\hline Mode & $\begin{array}{l}\text { Exp. / } \\
\mathrm{cm}^{-1}\end{array}$ & $\begin{array}{l}\text { Theor. / } \\
\mathrm{cm}^{-1}\end{array}$ & Assignments \\
\hline 42 & 3595 & 3436 & $v_{\mathrm{as}} \mathrm{N}_{10} \mathrm{H}_{13,14}[98.4]$ \\
\hline 41 & 3379 & 3394 & $v_{3} \mathrm{~N}_{3} \mathrm{H}_{16}[95.3]$ \\
\hline 40 & 3300 & 3340 & $\mathrm{vN}_{1} \mathrm{H}_{15}[95.0]$ \\
\hline 39 & 3230 & 3294 & $v_{90} \mathrm{~N}_{0} \mathrm{H}_{11,12}[65.0]+v_{5} \mathrm{~N}_{10} \mathrm{H}_{13,14}[34.3]$ \\
\hline 38 & 3177 & 3291 & $v_{s} \mathrm{~N}_{10} \mathrm{H}_{13,4}[58.7]+v_{a s} \mathrm{~N}_{9} \mathrm{H}_{11,12}[39.9]$ \\
\hline 37 & & 3235 & $v_{\mathrm{s}}^{\mathrm{s}} \mathrm{N}_{9} \mathrm{H}_{11,12}[98.0]$ \\
\hline 36 & 1647 & 1676 & $\begin{array}{l}\pi \mathrm{N}_{9} \mathrm{H}_{11,12}[58.7]+\delta \mathrm{N}_{10} \mathrm{H}_{13,14}[19.2]+ \\
v \mathrm{C}_{45}[10.5]+\delta \mathrm{C}_{2} \mathrm{~N}_{3} \mathrm{H}_{16}[8.7]\end{array}$ \\
\hline 35 & & 1632 & $\begin{array}{l}\pi \mathrm{N}_{9} \mathrm{H}_{11,12}[49.2]+\delta \mathrm{N}_{10} \mathrm{H}_{13,14}[37.3]+ \\
\delta \mathrm{C}_{2} \mathrm{~N}_{3} \mathrm{H}_{16}[6.9]+v \mathrm{C}_{4,5}[5.5]\end{array}$ \\
\hline 34 & 1565 & 1586 & $\begin{array}{l}\delta \mathrm{N}_{10} \mathrm{H}_{13,14}[42.9]+\pi \mathrm{N}_{9} \mathrm{H}_{11,12}[22.5]+ \\
\delta \mathrm{C}_{2} \mathrm{~N}_{3} \mathrm{H}_{16}[20.1]+v \mathrm{C}_{4,5}[10.4]\end{array}$ \\
\hline 33 & & 1574 & $\begin{array}{l}\left(\delta \mathrm{N}_{3} \mathrm{H}_{16}+\delta \mathrm{N}_{1} \mathrm{H}_{15}\right)[56.7]+\pi \mathrm{N}_{9} \mathrm{H}_{11,12}[13.7]+ \\
\delta \mathrm{N}_{10} \mathrm{H}_{13,14}[11.5]+v \mathrm{C}_{4,5}[10.1]+\left(\mathrm{v}_{2} \mathrm{~S}_{7}+\right. \\
\left.v \mathrm{C}_{6} \mathrm{~S}_{8}\right)[8.0]\end{array}$ \\
\hline 32 & 1462 & 1500 & $\begin{array}{l}\left(v \mathrm{C}_{2} \mathrm{~N}_{1}+\delta \mathrm{N}_{1} \mathrm{H}_{15}\right)[36.8]+\left(\mathrm{vC}_{4} \mathrm{~N}_{3}+\right. \\
\left.\delta \mathrm{N}_{3} \mathrm{H}_{16}\right)[23.0]+v \mathrm{C}_{5,6}[14.8]+ \\
\delta \mathrm{N}_{10} \mathrm{H}_{13,4}[15.4]+\pi \mathrm{N}_{9} \mathrm{H}_{11,12}[8.5]\end{array}$ \\
\hline 31 & 1430 & 1400 & $\begin{array}{l}v\left(\mathrm{C}_{4}-\mathrm{N}_{10} \mathrm{H}_{13,14}\right)[38.6]+v \mathrm{C}_{5,6}[25.7]+ \\
v \mathrm{C}_{2} \mathrm{~N}_{3}[20.8]+\delta \mathrm{N}_{1} \mathrm{H}_{15}[14.8]\end{array}$ \\
\hline 30 & 1386 & 1386 & $\begin{array}{l}\left(\delta \mathrm{N}_{1} \mathrm{H}_{15}+\delta \mathrm{N}_{3} \mathrm{H}_{16}\right)[57.1]+\mathrm{v}\left(\mathrm{C}_{4}-\mathrm{N}_{10} \mathrm{H}_{13,14}\right) \\
{[22.4]+\left(\mathrm{v}_{6} \mathrm{~S}_{8}+\mathrm{v} \mathrm{C}_{2} \mathrm{~S}_{7}\right)[11.8]+\mathrm{v}\left(\mathrm{C}_{5-}\right.} \\
\left.\mathrm{N}_{9} \mathrm{H}_{11,11}\right)[8.5]\end{array}$ \\
\hline 29 & 1300 & 1292 & $\begin{array}{l}\left(v \mathrm{~N}_{1} \mathrm{C}_{2} \mathrm{~N}_{3}+\delta \mathrm{N}_{3} \mathrm{H}_{16}\right)[36.1]+ \\
\pi \mathrm{N}_{9} \mathrm{H}_{41,12}[28.4]+\rho \mathrm{N}_{10} \mathrm{H}_{13,14}[17.8]+ \\
\mathrm{v}_{\mathrm{as}} \mathrm{C}_{4,5,6}[17.5]\end{array}$ \\
\hline 28 & 1276 & 1276 & $\begin{array}{l}v \mathrm{C}_{5} \mathrm{~N}_{9} \mathrm{H}_{11,12}[33.8]+\rho \mathrm{N}_{10} \mathrm{H}_{13,14}[28.1]+ \\
\left(v_{\mathrm{as}} \mathrm{C}_{2} \mathrm{~N}_{3} \mathrm{C}_{4}+\delta \mathrm{N}_{3} \mathrm{H}_{16}\right)[28.1]+\mathrm{vC}_{6} \mathrm{~N}_{1}[9.8]\end{array}$ \\
\hline 27 & 1232 & 1237 & $\begin{array}{l}\tau \mathrm{N}_{9} \mathrm{H}_{11,12}[76.6]+\left(v \mathrm{~N}_{1} \mathrm{C}_{2} \mathrm{~N}_{3}+\delta \mathrm{N}_{3} \mathrm{H}_{16}\right) \\
{[16.0]+\pi \mathrm{C}_{4,5,6}[7.2]}\end{array}$ \\
\hline 26 & 1182 & 1166 & $\begin{array}{l}\left(v \mathrm{~N}_{1} \mathrm{C}_{6}+\delta \mathrm{N}_{1} \mathrm{H}_{15}\right)[40.5]+\rho \mathrm{N}_{10} \mathrm{H}_{13,14}[22.6]+ \\
\left(v \mathrm{~N}_{3} \mathrm{C}_{2}+\delta \mathrm{N}_{3} \mathrm{H}_{16}\right)[17.9]+v \mathrm{~N}_{9} \mathrm{C}_{5}[17.8]\end{array}$ \\
\hline 25 & 1156 & 1142 & $\begin{array}{l}\left(\rho \mathrm{N}_{10} \mathrm{H}_{13,14}+\mathrm{C}_{4} \mathrm{~N}_{10}\right)[31.2]+\left(\mathrm{vC}_{2} \mathrm{~N}_{1} \mathrm{C}_{6}+\right. \\
\left.\left.\delta \mathrm{N}_{1} \mathrm{H}_{15}\right)[25.6]+\delta \mathrm{N}_{3} \mathrm{H}_{16}\right)[25.7]+ \\
\omega \mathrm{N}_{9} \mathrm{H}_{11,12}[13.8]\end{array}$ \\
\hline 24 & & 1062 & $\begin{array}{l}\rho \mathrm{N}_{10} \mathrm{H}_{13,14}[50.1]+\left(\delta \mathrm{N}_{13} \mathrm{H}_{16}+\delta \mathrm{N}_{1} \mathrm{H}_{15}\right) \\
{[22.7]+\left(\mathrm{vC}_{2} \mathrm{~S}_{7}+\mathrm{vC}_{6} \mathrm{~S}_{8}\right)[13.7]+} \\
\omega \mathrm{N}_{9} \mathrm{H}_{11,12}[8.2]+\mathrm{vC}_{4,5}[5.6]\end{array}$ \\
\hline 23 & 1015 & 1025 & $\begin{array}{l}v_{\mathrm{as}} \mathrm{C}_{2} \mathrm{~N}_{3} \mathrm{C}_{4}[29.2]+\mathrm{v}_{\mathrm{as}} \mathrm{C}_{4,5} \mathrm{~N}_{1}[24.7]+ \\
\omega \mathrm{N}_{9} \mathrm{H}_{11,12}[24.5]+\rho \mathrm{N}_{10} \mathrm{H}_{13.14}[21.6]\end{array}$ \\
\hline 22 & 910 & 942 & $\begin{array}{l}\omega \mathrm{N}_{9} \mathrm{H}_{11,12}[50.7]+v_{\mathrm{s}} \mathrm{N}_{1} \mathrm{C}_{2} \mathrm{~N}_{3}[23,7]+ \\
\rho \mathrm{N}_{10} \mathrm{H}_{13,14}[17,7]+\mathrm{v}_{\mathrm{s}} \mathrm{C}_{4,5,6}[7,7]\end{array}$ \\
\hline 21 & 863 & 902 & $\begin{array}{l}\omega \mathrm{N}_{9} \mathrm{H}_{11,12}[52.0]+v \mathrm{C}_{6} \mathrm{~S}_{8}[21.4]+ \\
\delta \mathrm{C}_{4} \mathrm{~N}_{3} \mathrm{C}_{2}[18.8]+\rho \mathrm{N}_{10} \mathrm{H}_{13.14}[7.7]\end{array}$ \\
\hline 20 & 746 & 745 & $\begin{array}{l}\left(\delta \mathrm{C}_{4,5,6}+\mathrm{vC}_{5} \mathrm{~N}_{9}\right)[38.1]+\delta_{\mathrm{s}} \mathrm{N}_{1} \mathrm{C}_{2} \mathrm{~N}_{3}[40.2]+ \\
\rho \mathrm{N}_{10} \mathrm{H}_{13,14}[21.5]\end{array}$ \\
\hline 19 & 712 & 727 & $\begin{array}{l}\pi \mathrm{N}_{1} \mathrm{H}_{15}[65.4]+\pi \mathrm{C}_{4,5,6}[23.6]+ \\
\tau \mathrm{N}_{3} \mathrm{H}_{16}[9.3]\end{array}$ \\
\hline 18 & 640 & 707 & $\begin{array}{l}\pi \mathrm{N}_{1} \mathrm{C}_{2} \mathrm{~N}_{3}[28.9]+\tau \mathrm{N}_{10} \mathrm{H}_{13,14}[26.2]+ \\
\pi \mathrm{C}_{4,5,6}[23.6]+\omega \mathrm{N}_{9} \mathrm{H}_{11,12}[21.1]\end{array}$ \\
\hline 17 & 623 & 645 & $\begin{array}{l}\tau \mathrm{N}_{10} \mathrm{H}_{13,14}[51.8]+\pi \mathrm{C}_{6} \mathrm{~N}_{1} \mathrm{C}_{5}[23.3]+ \\
\omega \mathrm{N}_{9} \mathrm{H}_{11,12}[16.1]+\pi \mathrm{C}_{4} \mathrm{~N}_{3} \mathrm{C}_{2}[8.8]\end{array}$ \\
\hline 16 & 600 & 618 & $\begin{array}{l}\pi \mathrm{N}_{1} \mathrm{C}_{2} \mathrm{~N}_{3}[45.3]+\tau \mathrm{N}_{10} \mathrm{H}_{13,14}[24.9]+ \\
\omega \mathrm{N}_{9} \mathrm{H}_{11,12}[15.1]+\pi \mathrm{C}_{4,5,6}[14.6]\end{array}$ \\
\hline 15 & 584 & 591 & $\begin{array}{l}\pi \mathrm{N}_{3} \mathrm{H}_{16}[33.4]+\tau \mathrm{N}_{10} \mathrm{H}_{13,14}[24.2]+ \\
\tau \mathrm{C}_{4,5,6}[18.7]+\tau \mathrm{N}_{1} \mathrm{H}_{15}[11.1]+ \\
\tau \mathrm{N}_{9} \mathrm{H}_{11,12}[7.5]\end{array}$ \\
\hline 14 & 571 & 570 & Sring $[85.0]+\pi \mathrm{N}_{3} \mathrm{H}_{16}[15.0]$ \\
\hline 13 & 545 & 563 & $\begin{array}{l}\pi \mathrm{N}_{3} \mathrm{H}_{16}[32.6]+\omega \mathrm{N}_{10} \mathrm{H}_{13,14}[29.4]+\left(\pi \mathrm{C}_{6} \mathrm{~S}_{8}+\right. \\
\left.\pi \mathrm{C}_{2} \mathrm{~S}_{7}\right)[11.1]+\omega \mathrm{N}_{9} \mathrm{H}_{11,12}[10.7]+\pi \\
\mathrm{N}_{1} \mathrm{H}_{15}[9.4] \\
\text { Sring }[81.9]+\pi \mathrm{N}_{3} \mathrm{H}_{16}[18.1]\end{array}$ \\
\hline
\end{tabular}

*Fractional composition of the vibrational modes are shown in parenthesis
Table 4. Experimental and theoretical vibrational spectrum of tautomer $\mathbf{3}^{*}$

\begin{tabular}{|c|c|c|c|}
\hline Mode & $\begin{array}{l}\text { Exp. / } \\
\mathrm{cm}^{-1}\end{array}$ & $\begin{array}{l}\text { Theor. / } \\
\mathrm{cm}^{-1}\end{array}$ & Assignments \\
\hline 42 & 3565 & 3490 & $\mathrm{v}_{\mathrm{as}} \mathrm{N}_{10} \mathrm{H}_{13,14}[95.0]$ \\
\hline 41 & 3379 & 3372 & $v \mathrm{~N}_{1} \mathrm{H}_{15}[92.4]+\mathrm{v}_{\mathrm{as}} \mathrm{N}_{10} \mathrm{H}_{13,14}[7.6]$ \\
\hline 40 & 3300 & 3345 & $v \mathrm{~N}_{1} \mathrm{H}_{15}[96.2]$ \\
\hline 39 & 3230 & 3288 & $v_{\text {as }} \mathrm{N}_{9} \mathrm{H}_{11,12}[99.3]$ \\
\hline 38 & 3177 & 3243 & $\mathrm{~V}_{\mathrm{s}} \mathrm{N}_{9} \mathrm{H}_{11,2}[98.8]$ \\
\hline 37 & 2577 & 2517 & $v \mathrm{~S}_{7} \mathrm{H}_{16}[99.3]$ \\
\hline 36 & 1647 & 1663 & $\begin{array}{l}\delta \mathrm{N}_{9} \mathrm{H}_{11,11}[62.8]+\delta \mathrm{N}_{10} \mathrm{H}_{13,14}[20.3]+ \\
v_{\mathrm{as}} \mathrm{C}_{4.5 .6}[9.5]+\mathrm{v}_{\mathrm{as}} \mathrm{N}_{1} \mathrm{C}_{2} \mathrm{~N}_{3}[7.3]\end{array}$ \\
\hline 35 & 1565 & 1628 & $\begin{array}{l}\left(\delta \mathrm{N}_{10} \mathrm{H}_{13,14}+v \mathrm{C}_{4} \mathrm{~N}_{10}\right)[57.6]+ \\
\delta \mathrm{N}_{9} \mathrm{H}_{11,12}[37.0]\end{array}$ \\
\hline 34 & 1525 & 1587 & $\begin{array}{l}\delta_{\mathrm{s}} \mathrm{N}_{10} \mathrm{H}_{13,14}[38.1]+\delta_{\mathrm{s}} \mathrm{N}_{9} \mathrm{H}_{11,12}[25.2]+ \\
\mathrm{v}_{\mathrm{as}} \mathrm{C}_{4,5,6}[12.8]+v_{\mathrm{as}} \mathrm{N}_{1} \mathrm{C}_{2} \mathrm{~N}_{3}[19.7]+ \\
\delta \mathrm{S}_{7} \mathrm{H}_{15}[4.7]\end{array}$ \\
\hline 33 & 1462 & 1542 & $\begin{array}{l}\pi \mathrm{N}_{1} \mathrm{H}_{15}[56]+v_{\text {as }} \mathrm{C}_{2} \mathrm{~N}_{3} \mathrm{C}_{4}[21.3]+ \\
\delta \mathrm{N}_{9} \mathrm{H}_{11,12}[17.1]+\delta \mathrm{N}_{10} \mathrm{H}_{13,14}[13.0]+ \\
\delta \mathrm{C}_{5} \mathrm{C}_{6} \mathrm{~S}_{8}[8.8]\end{array}$ \\
\hline 32 & 1430 & 1508 & $\begin{array}{l}\delta \mathrm{C}_{4,5,6}[40.0]+\delta \mathrm{N}_{1} \mathrm{H}_{15}[29.9]+\delta \mathrm{N}_{10} \mathrm{H}_{13,14}[22.2]+ \\
v \mathrm{~N}_{2} \mathrm{C}_{3}[7.8]\end{array}$ \\
\hline 31 & 1386 & 1460 & $\begin{array}{l}v\left(\mathrm{C}_{4}-\mathrm{N}_{10} \mathrm{H}_{13,14}\right)[67.7]+v_{\text {as }} \mathrm{N}_{1} \mathrm{C}_{2} \mathrm{~N}_{3}[16.5]+ \\
\delta \mathrm{N}_{9} \mathrm{H}_{11,12}[10.8]+v \mathrm{C}_{5,6}[5.7]\end{array}$ \\
\hline 30 & 1346 & 1340 & $\begin{array}{l}\mathrm{vC}_{4,5,6}[24.5]+\delta \mathrm{N}_{1} \mathrm{H}_{15}[22.5]+\tau \mathrm{N}_{9} \mathrm{H}_{11,12}[22.1]+ \\
\delta \mathrm{N}_{3} \mathrm{C}_{2} \mathrm{~S}_{8} \mathrm{H}_{16}[18.3]+\rho \mathrm{N}_{10} \mathrm{H}_{13,14}[12.8]\end{array}$ \\
\hline 29 & 1300 & 1285 & $\begin{array}{l}\left(\mathrm{vC}_{5} \mathrm{~N}_{9}+\delta \mathrm{N}_{9} \mathrm{H}_{11,12}\right)[33.0]+\rho \mathrm{N}_{10} \mathrm{H}_{13,14}[24.6]+ \\
v_{\text {as }} \mathrm{C}_{2} \mathrm{~N}_{3} \mathrm{C}_{4}[16.4]+\delta \mathrm{N}_{1} \mathrm{H}_{15}[15.9]+ \\
\mathrm{vC}_{6} \mathrm{~S}_{8}[5.5]\end{array}$ \\
\hline 28 & 1276 & 1233 & $\begin{array}{l}\tau \mathrm{N}_{9} \mathrm{H}_{11,12}[67.0]+\rho \mathrm{N}_{10} \mathrm{H}_{13,14}[12.3]+ \\
\delta \mathrm{N}_{1} \mathrm{H}_{15}[8.0]+\delta \mathrm{C}_{2} \mathrm{~S}_{7} \mathrm{H}_{16}[4.8]\end{array}$ \\
\hline 27 & 1232 & 1211 & $\begin{array}{l}\rho \mathrm{N}_{10} \mathrm{H}_{13,14}[33.5]+\tau \mathrm{N}_{9} \mathrm{H}_{11,12}[25.1]+ \\
\pi \mathrm{N}_{1} \mathrm{H}_{15}[20.9]+\delta \mathrm{C}_{2} \mathrm{~N}_{3} \mathrm{C}_{4}[9.4]+\delta \mathrm{S}_{7} \mathrm{H}_{16}[4.8]\end{array}$ \\
\hline 26 & 1182 & 1142 & $\begin{array}{l}\left(v \mathrm{v}_{6} \mathrm{~N}_{1}+\delta \mathrm{N}_{1} \mathrm{H}_{15}\right)[53.1]+\left(\mathrm{vC}_{5} \mathrm{~N}_{9}+\right. \\
\left.v \mathrm{~N}_{9} \mathrm{H}_{11,12}\right)[26.6]+\delta \mathrm{S}_{7} \mathrm{H}_{16}[10.6]+ \\
v \mathrm{C}_{2} \mathrm{~N}_{3} \mathrm{C}_{4}[7]\end{array}$ \\
\hline 25 & 1156 & 1071 & $\begin{array}{l}\rho \mathrm{N}_{10} \mathrm{H}_{13,14}[46.1]+\delta \mathrm{N}_{1} \mathrm{H}_{15}[17.9]+ \\
\mathrm{vC}_{2} \mathrm{~N}_{3} \mathrm{C}_{4}[11.5]+\mathrm{vC}_{6} \mathrm{~S}_{8}[6.3]+\left(\mathrm{vC}_{5} \mathrm{~N}_{9}+\right. \\
\left.\omega \mathrm{N}_{9} \mathrm{H}_{11,12}\right)[14.0]\end{array}$ \\
\hline 24 & 1015 & 1040 & $\begin{array}{l}\rho \mathrm{N}_{10} \mathrm{H}_{13,14}[24.8]+\omega \mathrm{N}_{9} \mathrm{H}_{11,12}[22.8]+\delta \mathrm{N}_{3} \mathrm{C}_{4} \mathrm{C}_{5} \\
{[20.0]+\delta \mathrm{C}_{2} \mathrm{~N}_{1} \mathrm{C}_{6}[16.8]+\delta \mathrm{S}_{7} \mathrm{H}_{16}[15.4]}\end{array}$ \\
\hline 23 & 910 & 959 & $\begin{array}{l}\delta \mathrm{S}_{7} \mathrm{H}_{16}[44.6]+\omega \mathrm{N}_{9} \mathrm{H}_{11,12}[29.1]+\rho \mathrm{N}_{10} \mathrm{H}_{13,14} \\
{[11.7]+v \mathrm{C}_{2} \mathrm{~N}_{1} \mathrm{C}_{6}[10.1]}\end{array}$ \\
\hline 22 & 863 & 935 & $\begin{array}{l}\omega \mathrm{N}_{9} \mathrm{H}_{11,12}[53.6]+\left(\delta \mathrm{S}_{7} \mathrm{H}_{16}+v \mathrm{~N}_{1} \mathrm{C}_{2} \mathrm{~N}_{3}\right)[32.9] \\
+\rho \mathrm{N}_{10} \mathrm{H}_{13,14}[8.0]\end{array}$ \\
\hline 21 & 806 & 885 & $\begin{array}{l}\omega \mathrm{N}_{9} \mathrm{H}_{11,12}[44.1]+\delta \mathrm{S}_{7} \mathrm{H}_{16}[24.5]+ \\
v \mathrm{C}_{2} \mathrm{~N}_{3} \mathrm{C}_{4}[21.3]+\mathrm{vC}_{6} \mathrm{~S}_{8}[9.9]\end{array}$ \\
\hline 20 & 746 & 752 & $\begin{array}{l}\pi \mathrm{N}_{3} \mathrm{C}_{4} \mathrm{C}_{5}[40,2]+\pi \mathrm{C}_{2} \mathrm{~N}_{1} \mathrm{C}_{6}[33.1]+ \\
\tau \mathrm{N}_{9} \mathrm{H}_{11,12}[25.7]\end{array}$ \\
\hline 19 & 712 & 725 & $\begin{array}{l}\tau \mathrm{N}_{10} \mathrm{H}_{13,14}[30.2]+v \mathrm{C}_{5} \mathrm{~N}_{9}[25.5]+ \\
\delta \mathrm{N}_{1} \mathrm{C}_{6} \mathrm{~S}_{8}[23.6]+v \mathrm{C}_{2} \mathrm{~S}_{7}[10]+\mathrm{vC}_{4} \mathrm{~N}_{3}[10.0]\end{array}$ \\
\hline 18 & & 672 & $\begin{array}{l}\pi \mathrm{N}_{1} \mathrm{H}_{15}[56.7]+\tau \mathrm{N}_{10} \mathrm{H}_{13,14}[11.8]+ \\
\pi \mathrm{C}_{4,5,6}[11.2]+\pi \mathrm{S}_{7} \mathrm{H}_{16}[11]+\omega \mathrm{N}_{9} \mathrm{H}_{11,12}[8.7]\end{array}$ \\
\hline 17 & & 646 & $\begin{array}{l}\tau \mathrm{N}_{10} \mathrm{H}_{13,14}[58.5]+\pi \mathrm{N}_{1} \mathrm{C}_{2} \mathrm{~N}_{3}[18,1]+ \\
\omega \mathrm{N}_{9} \mathrm{H}_{11,12}[13.9]+\pi \mathrm{C}_{4,5,6}[9.3]\end{array}$ \\
\hline 16 & 600 & 638 & $\begin{array}{l}\tau \mathrm{N}_{10} \mathrm{H}_{13,14}[44.1]+\pi \mathrm{N}_{1} \mathrm{C}_{2} \mathrm{~N}_{3}[30.3]+ \\
\pi \mathrm{C}_{4,5,6}[25.8]\end{array}$ \\
\hline 15 & & 592 & $\begin{array}{l}\left(\pi \mathrm{N}_{1} \mathrm{H}_{15}+\pi \mathrm{N}_{1} \mathrm{C}_{5} \mathrm{C}_{6}\right)[65.8]+\pi \mathrm{C}_{4} \mathrm{~N}_{3} \mathrm{C}_{2}[28.3]+ \\
\pi \mathrm{S}_{7} \mathrm{H}_{16}[5.8]\end{array}$ \\
\hline 14 & 571 & 569 & $\begin{array}{l}v \text { ring }[41.1]+\rho \mathrm{N}_{10} \mathrm{H}_{13,14}[33.4]+ \\
\omega \mathrm{N}_{9} \mathrm{H}_{11,12}[17.4]+\delta \mathrm{S}_{7} \mathrm{H}_{16}[7.9]\end{array}$ \\
\hline 13 & 545 & 540 & $\begin{array}{l}\rho \mathrm{N}_{10} \mathrm{H}_{13,14}[36.9]+\delta \operatorname{ring}[23.1]+ \\
\omega \mathrm{N}_{9} \mathrm{H}_{11,12}[18.0]+\delta \mathrm{N}_{1} \mathrm{H}_{15}[14.7]+ \\
\delta \mathrm{S}_{7} \mathrm{H}_{16}[7.2]\end{array}$ \\
\hline 12 & 495 & 442 & $\begin{array}{l}\text { Sring }[55.9]+\mathrm{vC}_{6} \mathrm{~S}_{8}[16,0]+\delta \mathrm{N}_{1} \mathrm{H}_{15}[14.8] \\
+\delta \mathrm{S}_{7} \mathrm{H}_{16}[13.0]\end{array}$ \\
\hline
\end{tabular}

*Fractional composition of the vibrational modes are shown in parenthesis 
dithiol structure. The characteristic $\mathrm{v}(\mathrm{N}-\mathrm{H})$ vibrational peaks expected for this isomer at 3521, 3387, 3319 at $3261 \mathrm{~cm}^{-1}$ (Table 2), practically coincide with the experimental bands observed at $3565,3379,3300$ and $3230 \mathrm{~cm}^{-1}$ for DADMcP. The $v(\mathrm{~S}-\mathrm{H})$ vibrations appear as weak bands in the theoretical spectrum of isomer 1, at 2678 and $2662 \mathrm{~cm}^{-1}$ (about $2 \%$ relative intensity), corresponding to the weak shoulders observed at 2693 and $2577 \mathrm{~cm}^{-1}$, respectively.

In the case of the tautomer 2 , which corresponds to the dithione form, the $\mathrm{v}(\mathrm{N}-\mathrm{H})$ vibrations are very close to those of tautomer $\mathbf{1}$, however there is no $(\mathrm{S}-\mathrm{H})$ band (Table 3$)$. Unfortunately, in this case, the $\mathrm{v}(\mathrm{C}=\mathrm{S})$ vibration does not correspond to a characteristic peak, since it always occurs in combination with $v(\mathrm{C}-\mathrm{N})$ and $v(\mathrm{C}-\mathrm{C})$ vibrations, e.g. at $1294 \mathrm{~cm}^{-1}$, in Table 2 .

In the case of tautomer $\mathbf{3}$, because of its mixed thiol and thione features, its theoretical vibrational spectrum exhibits a close similarity with those of tautomers $\mathbf{1}$ and 3, as shown in Table 4.

Therefore, one can see that the infrared spectra fit fairly well those predicted by the theoretical model, corroborating the existence of a mixture of tautomers.

Additional evidence for the occurrence of the thiol tautomers ( $\mathbf{1}$ or $\mathbf{3}$ ) was obtained from the near-infrared (NIR) spectrum of the DADMcP species, as shown in Figure 4. In fact, it should be mentioned that the NIR region is being increasingly exploited in analytical chemistry, because of the occurrence of characteristic overtone and combination bands of the $\mathrm{C}-\mathrm{H}, \mathrm{N}-\mathrm{H}, \mathrm{O}-\mathrm{H}$ and $\mathrm{S}-\mathrm{H}$ fundamental vibrations. In the DADMcP compound, only the $\mathrm{N}-\mathrm{H}$ and $\mathrm{S}-\mathrm{H}$ vibrations ${ }^{30}$ can contribute to the NIR spectrum, occurring at frequencies approximately two times those observed for the fundamental ones in the middle IR region, i.e., at 6653, 6473, 6293 and 4968, $4778 \mathrm{~cm}^{-1}$, respectively (Figure 4).

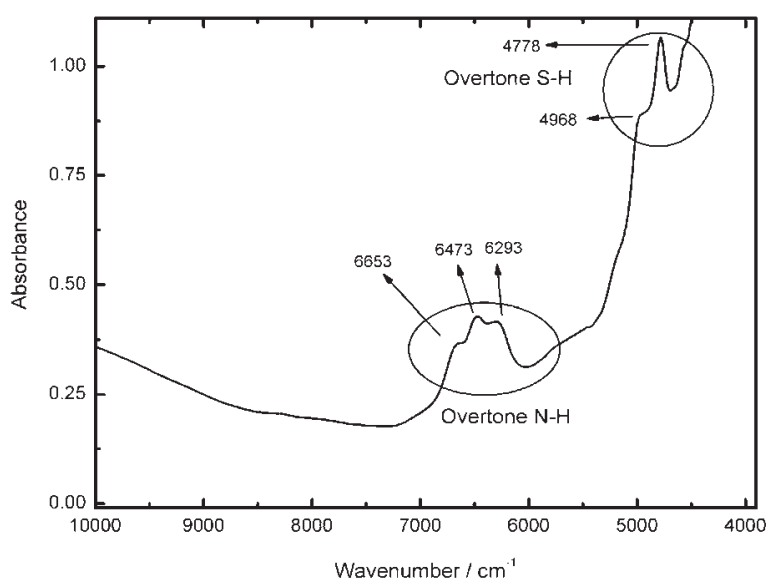

Figure 4. NIR spectrum of 4,5-diamine-2,6-dimercaptopyrimidine in solid form.

\section{Conclusions}

Due to the thione-thiol equilibrium, the 4,5-diamine2,6-dimercaptopyrimidine compound can exhibit nine tautomeric forms. HF/SCF and DFT calculations support the existence of at least two major tautomeric forms of which the dithione tautomer is the most stable one. A successful simulation of the vibrational spectra of the most stable tautomers is reported, showing a good agreement with the experimental bands in the FTIR spectrum. According to the theoretical and experimental evidence, tautomers $\mathbf{2}$ and $\mathbf{3}$ should be the predominant species in the solid state. This conclusion is corroborated by the $\mathrm{S}-\mathrm{H}$ overtone band, in the NIR spectrum.

\section{Acknowledgments}

The support from FAPESP, CNPq, and IM2C is gratefully acknowledged.

\section{References}

1. Senge, M. O.; Angew. Chem. Int. Ed. 1996, 35, 1923.

2. Raper, E. S.; Coord. Chem. Rev. 1996, 153, 199.

3. Fandos, R.; Lafranchi, A.; Otero, A.; Pellinghelli, M. A.; Ruiz, M. J.; Terreros, P.; Organometallics 1996, 15, 4725.

4. Brandt, K.; Sheldrick, W. S.; Inorg. Chim. Acta 1998, 267, 39.

5. Raper, E. S.; Coord. Chem. Rev. 1996, 165, 475.

6. Spinner, E.; J. Chem. Soc. 1960, 1237.

7. Stoyanov, S.; Petrov, I.; Antonov, L.; Stoyanov, T.; Karagiannidis, P.; Aslanidis, P.; Can. J. Chem. 1990, 68, 1482.

8. Stoyanov, S.; Stoyanov, T.; Akrivos, P. D.; Trends Appl. Spectrosc. 1998, 2, 89.

9. Chou, P. T.; Wei, C. Y.; Hung, F. T.; J. Phys. Chem. B 1997, 101, 9119.

10. Beak, P.; Fry, F. S.; Lee Jr., J.; Steele, F.; J. Am. Chem. Soc. 1976, 98, 171.

11. Matsuda, Y.; Ebata, T.; Mikami, N.; J. Phys. Chem. A 2001, 105,3475 .

12. Kwiatkowski, J. S.; Leszczynski, J.; J. Mol. Struct. 1996, 376, 325 .

13. Cakir, S.; Bicer, E.; Odabasoglu, M.; Albayrak, C.; J. Braz. Chem. Soc. 2005, 16, 711.

14. Wong, M. W.; Wiberg, K. B.; Frisch, M. J.; J. Am. Chem. Soc. 1992, 114, 1645.

15. Schlegel, H. B.; Gund, P.; Fluder, E. M.; J. Am. Chem. Soc. 1982, 104, 5347.

16. Moran, D.; Sukcharoenphon, K.; Puchta, R.; Schaefer III, H. F.; Schleyer, P. V. R.; Hoff, C. D.; J. Org. Chem. 2002, 67, 9061.

17. Lorenc, J.; Dyminska, L.; Mohmed, A. F. A.; Hanuza, J.; Talik, Z.; Maczka, M.; Macalik, L.; Chem. Phys. 2007, 334, 90. 
18. Hatherley, L. D.; Brown, R. D.; Godfrey, P. D.; Pierlot, A. P.; Caminati, W.; Damiani, D.; Melandri, S.; Favero, L. B.; J. Phys. Chem. 1993, 97, 46

19. Wang, J. H.; Boyd, R. J.; J. Phys. Chem. 1996, 100, 16141.

20. Demets, G. J. F.; PhD Thesis, Universidade de São Paulo, Brazil, 2001.

21. Bonacin. J. A.; Formiga, A. L. B.; Melo, V. H. S.; Toma, H. E.; Vib. Spectrosc. 2007, 44, 133.

22. Schmidt, M. W.; Baldridge, K. K.; Boatz, J. A.; Elbert, T. S.; Gordon, M. S.; Jensen, J. H.; Koseki, S.; Matsunaga, N.; Nguyen, K. A.; Su, S.; Windus, T. L.; Dupuis, M., Montgomery, Jr., J. A.; J. Comput. Chem. 1993, 14, 1347.

23. Becke, A. D.; J. Chem. Phys. 1993, 98, 5648.

24. Lee, C.; Yang, W.; Parr, R. G.; Phys. Rev. B 1988, 37, 785.
25. Miehlich, B.; Savin, M. A.; Stoll, H.; Chem. Phys. Let. 1989, 157, 200.

26. Scott, A. P.; Radom, L.; J. Phys. Chem. 1996, 100, 16502.

27. Gorelsky, S. I.; Lever, A. B. P.; Can. J. Anal. Sci. Spectrosc. 2003, 48, 93.

28. Estrin, D. A.; Paglieri, L.; Corongiu, G.; J. Phys. Chem. 1994, 98, 5653.

29. Tian, S. X.; Xu, K. Z.; Chem. Phys. 2001, 264, 187.

30. Goddu, R. F.; Delker, D. A.; Anal. Chem. 1960, 32, 140.

Received: October 9, 2007 Published on the web: February 22, 2008

FAPESP helped in meeting the publication costs of this article. 